\title{
ROBUST CHEMICAL SOLVER FOR FULLY-IMPLICIT SIMULATIONS
}

\author{
COLIN MCNEECE, XAVIER RAYNAUD, HALVOR NILSEN, AND MARC HESSE
}

\begin{abstract}
The study of geological systems requires the solution of complex geochemical relations. We present an implementation of a chemical solver which can handle various types of models, including surface chemistry. The implementation is done in view of easy coupling with flow simulations to obtain a fully-coupled, fully-implicit solver for chemical reaction transport equations applicable to realistic reservoir models.
\end{abstract}

\section{INTRODUCTION}

In this paper, we present simulation and implementations strategies for computing solutions of a variety of chemical models. The solver is open-source and available for download [7]. The implementation supports non-isothermal multicomponent aqueous complexation, surface complexation (including the CD-MUSIC model), ion exchange, and dissolution/precipitation. After rewriting the standard chemical equilibrium in a generic form, we explain how a standard log-log transformation of the residuals and primary variables can improve the robustness of the Newton method. We derive simple apriori bounds on the system unknowns and show numerically how they contribute to improve the convergence. Our solving strategy includes the computation of good initial guesses calculated explicitly by sequentially adding model complexity.

The implementation relies heavily on automatic differentiation which eliminates the burden of computing analytically and implementing the jacobians of the residuals. We also take profit of an existing platform to assemble the equations and run the Newton steps. We use open-source MRST and refer to [4] for detailed explanation on how the tool can be used in this setting. More significantly, MRST offers supports for unstructured grids which are typically associated with realistic reservoir models. Discrete differentiation operators for finite volume methods are readily available. The discrete differential operators and automatic differentiation are the two ingredients which significantly simplify the implementation of fullyimplicit multi-physics solvers for reservoir flow simulations. Prototyping allows for testing of solver strategies and, once the best methods are identified, computational speed can be optimized, possibly by switching to a compiled language.

The chemical solver has been validated for each model against PHREEQC [9] which is the reference chemical solver for applications in geochemistry. The benchmark tests are not included in this paper but are available in the manual which can be found in [7].

The coupled chemistry-transport equations are presented here and the simulation of these equations, using fully-coupled and fully-implicit methods, is clearly 
one of the main motivations behind the implementation of the chemical solver we have presenting here. Still, at the moment of publication, we only have one-sided coupled simulations running, that is, simulations where the chemical composition has no effect on the flow. We are working on a fully coupled test, in our case, a carbon system which includes the dissolution of calcite and thus the increase of permeability. We plan to release results in a very near future. Different numerical schemes (for example splitting strategies) and additional physics can also be incorporated into the existing tools through the object-oriented framework employed by MRST.

Identifying the generic structure of chemical equations allows us to develop a flexible user interface. The solver can handle arbitrarily complex geochemical systems with any choice of species or element concentration as input depending on what is known of the chemical system. The user interface is essentially based on parsing of input strings and the string inputs use standard notations in chemistry.

\section{Modeling equations}

2.1. General structure of the equilibrium equations. In this section, we present a brief derivation of the equilibrium equations with an emphasis on the algebraic structure of the equations. We remark that the system of equations split into two sets: A first set of equations which are linear in the logarithm of the concentrations and a second set where the equations are linear with respect to the concentration. In the following section, this structure is used to justify the robustness of the Newton solver when using a log-log transformation.

Let us consider $n_{c}$ chemical species, $\left\{C_{i}\right\}_{i=1}^{n_{c}}$, in quantities $\left\{N_{i}\right\}_{i=1}^{n_{c}}$, entering into $n_{e}$ chemical reactions. The stochiometric relations can be written (by moving all terms to the left side, for notational convenience):

$$
a_{k, 1} C_{1}+a_{k, 2} C_{2}+\ldots+a_{k, n_{c}} C_{n_{c}} \stackrel{K_{k}^{+}}{\underset{K_{k}^{-}}{\rightleftarrows}} 0,
$$

where $a_{k, i}$ are integers which can also be negative numbers. Let us consider a variation $\delta N$ in the quantities of components. This variation is a result of the chemical reactions. Let $\nu_{k}$ be the number of times the reaction $k$ has occurred. We have

$$
\delta N=-\sum_{k=1}^{n_{e}} \nu_{k} a_{k}=-A^{t} \nu
$$

where $a_{k}$ denotes the row of $A$ and $\nu=\left(\nu_{1}, \ldots, \nu_{n_{e}}\right)$. Hence, $\delta N \in \operatorname{rank}\left(A^{t}\right)$, that is, $\delta N \in \operatorname{ker}(A)^{\perp}$. Let us $V$ denotes a basis of $\operatorname{ker}(A)$ and $W$ a basis of its complement. Provided that there are no redundancies in the chemical reactions described by $A$, its rows will be linearly independent, and we can (for instance) choose $W=A^{t}$. From (2), we have

$$
V^{t} \delta N=0
$$

which shows that the kernel of $A^{t}$ identifies natural linear combinations that are preserved at equilibrium. To see this more clearly, let us consider the kinetic equations, before the equilibrium is reached. For every chemical reaction, there corresponds the kinetic equation given by

$$
\frac{d N_{i}}{d t}=F_{i}
$$


where $F_{i}$ is defined as

$$
F_{i}=-\sum_{k=1}^{n_{e}} a_{k, i} G_{k}=-A^{t} G
$$

for

$$
G_{k}=k_{k}^{+} \prod_{a_{k, i>0}} N_{i}^{\left|a_{k, i}\right|}-k_{k}^{-} \prod_{a_{k, i<0}} N_{i}^{\left|a_{k, i}\right|}
$$

and $G=\left(G_{1}, \ldots, G_{n_{e}}\right)$. The definition of $G_{k}$ is valid if all components which enter the chemical equation $k$ have activities equal to their concentration, which we assume for now (it is not always true, e.g. for $\mathrm{H}_{2} \mathrm{O}$ or for precipitated substances such as calcite). Let

$$
M=V^{t} N \quad \text { and } \quad P=W^{t} N .
$$

The governing equations (4) can then be decomposed in two sets such that

$$
\frac{d}{d t} M=0 \quad \text { and } \quad \frac{d}{d t} P=W^{t} F .
$$

This decomposition shows that the linear combinations given by the kernel basis $V$ are invariant quantities. We do not need such mathematical approach to establish the existence of invariant quantities. Indeed, in the system we are considering, the chemical species are molecules, meaning that they correspond to a given combination of atoms. The atoms are constitutive elements and the total number of each type of atom is preserved. The concentration of a given atom is obtained as a linear combination of the concentration, weighted by the occurrence number of the atom in the molecules. Note that, in the presentation above, we did not mention atoms and only the species, see (1). Thus, we may wonder how, ignoring the existence of atoms, we managed to infer the existence of invariants, which in turn correspond to the atoms. This is explained by the fact that the chemical reaction equations are always well-balanced in the sense that each of them, when written explicitly, have preserved quantities, which precisely correspond to the atoms involved in the composition of the molecules entering the equation. We recover these invariants, in a purely algebraic manner, by looking at the kernel of the reaction matrix $A$. However, the atomic nature of the species gives us a precious additional structure which turns to be determinant in the solution procedure we will describe later. In the previous algebraic derivation, the matrix $V$ is not unique and we have no result on the sign of the coefficients of $V$. But now, by using the distribution of each atom into the species, we know that we can find a matrix $V$ such that all the coefficients of $V$ are non-negative, see (17) in the illustrative example below. There exist a well-known invariant of the reaction equations: Charge balance. The charge balance, as the conservation of atom type, is also explicitly enforced in every reaction equation. However the decomposition of the charge in terms of the species does not lead to a linear combinations with only non-negative elements, see (18) in the example.

From (8), we infer that the chemical equilibrium equation are given by $W^{t} F=0$, that is,

$$
W^{t} A^{t} G=0 .
$$


If we can choose $W=A^{t}$, this equation reduces to $A A^{t} G=0$. Since $W^{t} A^{t}$ is invertible, and from (9), we recover as expected that the chemical equilibrium equations are given by $G=0$.

Let us simplify the notations and denote by $x \in \mathbb{R}^{n_{c}}$ the vector of species concentrations (denoted previously $N$ ) and $\hat{x} \in \mathbb{R}^{n_{c}}$ the vector of the logarithm of the concentrations, that is $\hat{x}_{i}=\ln \left(x_{i}\right)$. At chemical equilibrium, we have $G=0$, which is equivalent to

$$
\prod_{j=1}^{n_{c}} x_{j}^{a_{k, j}}=K_{k}
$$

for $K_{k}=\frac{K_{k}^{+}}{K_{k}^{-}}$. Equation (10) is equivalent to

$$
\sum_{j=1}^{n_{c}} a_{k, j} \hat{x}_{j}=\hat{K}_{k}
$$

where $\hat{K}_{k}=\ln \left(K_{k}\right)$. We introduce the concentration $X_{i}$ of each atom or constitutive element. By looking at the occurrence of a given atom in each species, we can assemble a matrix $V$ such that

$$
\sum V_{j, i} x_{j}=X_{i}
$$

The equilibrium equations consist of (11) and (12), which we can rewrite synthetically as

$$
\begin{aligned}
A \hat{x} & =\hat{K}, \\
V^{t} x & =X .
\end{aligned}
$$

Thus, the chemical equilibrium equations consist of a set of linear equations of either the concentrations or the logarithm of the concentration. Moreover, the set of equations that corresponds to the linear combinations of the concentrations only involve positive coefficients.

2.2. Illustrative example of a carbon system. We consider a carbon system. The reactions are the following

$$
\begin{aligned}
& \mathrm{H}_{2} \mathrm{O} \rightleftarrows \mathrm{H}^{+}+\mathrm{OH}^{-}, \\
& \mathrm{H}_{2} \mathrm{CO}_{3} \rightleftarrows \mathrm{H}^{+}+\mathrm{HCO}_{3}^{-}, \\
& \mathrm{HCO}_{3}^{-} \rightleftarrows \mathrm{H}^{+}+\mathrm{CO}_{3}^{2-} .
\end{aligned}
$$

There are $n_{e}=3$ reactions and $n_{c}=9$ components, which we order as follows,

$$
\mathrm{H}_{2} \mathrm{O}, \mathrm{H}^{+}, \mathrm{OH}^{-}, \mathrm{H}_{2} \mathrm{CO}_{3}, \mathrm{HCO}_{3}^{-}, \mathrm{CO}_{3}^{2-} \text {. }
$$

The matrix $A$, as defined in the previous section, is given by

$$
A=\left(\begin{array}{cccccc}
1 & -1 & -1 & 0 & 0 & 0 \\
0 & -1 & 0 & 1 & -1 & 0 \\
0 & -1 & 0 & 0 & 1 & -1
\end{array}\right)
$$

The rank of $A$ is 3 . We can obtain a basis of $\operatorname{ker}(A)$ by considering the total conservation of each constitutive element, which are in this case $\mathrm{C}, \mathrm{O}, \mathrm{H}$. The total conservation of $\mathrm{C}$ gives

$$
\delta\left(\left[\mathrm{H}_{2} \mathrm{CO}_{3}\right]+\left[\mathrm{HCO}_{3}^{-}\right]+\left[\mathrm{CO}_{3}^{2-}\right]\right)=0,
$$


the total conservation of $\mathrm{O}$ gives

$$
\delta\left(m\left(\mathrm{H}_{2} \mathrm{O}\right)+\left[\mathrm{OH}^{-}\right]+3\left[\mathrm{H}_{2} \mathrm{CO}_{3}\right]+3\left[\mathrm{HCO}_{3}^{-}\right]+3\left[\mathrm{CO}_{3}^{2-}\right]\right)=0
$$

and the total conservation of $\mathrm{H}$ gives

$$
\delta\left(2 m\left(\mathrm{H}_{2} \mathrm{O}\right)+\left[\mathrm{H}^{+}\right]+\left[\mathrm{OH}^{-}\right]+2\left[\mathrm{H}_{2} \mathrm{CO}_{3}\right]+\left[\mathrm{HCO}_{3}^{-}\right]\right)=0 .
$$

Thus, we obtain the matrix

$$
V=\left(\begin{array}{lll}
0 & 1 & 2 \\
0 & 0 & 1 \\
0 & 1 & 1 \\
1 & 3 & 2 \\
1 & 3 & 1 \\
1 & 3 & 0
\end{array}\right)
$$

and we can check directly that $A V=0$. Moreover, all the coefficients of $V$ are positive, which is expected as the matrix was assembled using the conservation of the constitutive elements entering the reactions. In comparison, the linear combination for the conservation of charge given by

$$
\delta\left(\left[\mathrm{H}^{+}\right]-\left[\mathrm{OH}^{-}\right]-\left[\mathrm{HCO}_{3}^{-}\right]-2\left[\mathrm{CO}_{3}^{2-}\right]\right)=0,
$$

does not have this property. Note that it can be recovered as a linear combination of the columns of $V$,

$$
e=\left(\begin{array}{r}
0 \\
1 \\
-1 \\
0 \\
-1 \\
-2
\end{array}\right)=4 V_{1}-2 V_{2}-V_{3}
$$

2.3. Activity of Aqueous Species. We relax the assumption of an ideal solution, and implement activities for all aqueous species. We denote by $\gamma_{j}$ the activity of the species index by $j$. When we consider activities, the equilibrium equation (10) are replaced by

$$
\prod_{j=1}^{n_{c}}\left(x_{j} \gamma_{j}\right)^{a_{k, j}}=K_{k} .
$$

After applying the logarithm, we get

$$
\sum_{j=1}^{n_{c}} a_{k, j}\left(\hat{x}_{j}+\hat{\gamma}_{j}\right)=\hat{K}_{k},
$$

where $\hat{\gamma}_{j}=\ln \left(\gamma_{j}\right)$. We can rewrite (20) in a condensed form, similar to (13a),

$$
A \hat{x}+A \hat{\gamma}=\hat{K} \text {. }
$$

The activity of aqueous species is determined by the extended Davies equation

$$
\log _{10}\left(\gamma_{j}\right)=A z_{i}^{2}\left(\frac{I^{1 / 2}}{1+I^{1 / 2}}-0.3 I\right)
$$

where $z_{j}$ is the charge of species $j$, and $I$ is the ionic strength of the bulk solution. The parameter $A$ being determined by

$$
A=1.82 \times 10^{6}\left(e_{w} T\right)^{-3 / 2} .
$$


where $e_{w}$ is the relative permeability of water, and $T$ is the temperature of the bulk solution (K) [1]. The dielectric constant of water is determined by the polynomial function [6]

$e_{w}=87.740-0.4008(T-273.15)+9.398 \times 10^{-4}(T-273.15)^{2}-1.410 \times 10^{-6}(T-273.15)^{3}$.

The ionic strength of the solution is calculated by

$$
I=\frac{1}{2} \sum_{j=1}^{n_{c}} z_{j} C_{j} \delta_{j}
$$

where, in this case, $\delta$ removes the contribution of charge from surface species and the electron $\mathrm{e}^{-}$, that is $\delta_{i}=0$ if species $i$ is on a surface or is $\mathrm{e}^{-}$and $\delta_{i}=1$ otherwise.

2.4. Surface chemistry. Many natural aqueous systems are composed of a liquid water phase in contact with a solid phase, such as a groundwater aquifer and the material that compose the subsurface. As the solid is composed of repeating chemical structures, the boundaries of the solid mark the location of under-coordinated atoms. These truncations in the crystal structure lead to charge accumulation at the interface of the solid and liquid phases. To balance this charge, ions and polar molecules in the liquid water phase migrate to, and interact with the solid surface. These chemical interactions can be long lived, forming covalent bonds, eventually leading to the precipitation of a further solid phase. Or, can be short lived, transient reactions, being driven by van der Waal type forces. In the present study, we model the later and will refer to them as sorption reactions.

In general all surface chemistry models define a surface as a finite number of sites with which chemical species in the liquid may interact. Depending on the model, the surfaces can be either occupied or free. However, in the case of the ion exchange model, a surface site must always be occupied; the sorption of one ion from the liquid necessitating the release of a sorbed ion. As with chemical reactions in the liquid, surface reactions are governed by laws of mass action, and conservation of surface sites. Practically, the main characteristic which mathematically distinguish surface reactions from aqueous reactions are the equations of state which govern the activity of surface species. Many mathematical models have been developed to capture this phenomenon both empirically, and mechanistically. The solver developed here is capable of solving the most common model forms, which are detailed below.

2.4.1. Activity Coefficients of Surface Species. The activity coefficients of surface species depends on the surface chemistry model that is employed.

The activity coefficients of species associated with ion exchange and Langmuir type surfaces is unity, and their concentrations are determined by their active fraction consistent with the Gaines-Thomas convention [2].

A more mechanistic approach to surface chemistry modeling takes the form of the so called surface complexation models. Such models represent the mineralliquid interface as capacitors in series, the interfaces of those layers being planes of charge (ion) accumulation. The activity coefficients of species associated with an electrostatic surface (such as the triple layer and constant capacitance models) are determined by the potential and charge of the planes which sorbing the species occupy,

$$
\gamma_{j}=\exp \left(\frac{F \sum_{p=1}^{n_{p}} \zeta_{j, o, p} \Psi_{o, p}}{R T}\right),
$$


where $F$ is Faraday's constant, $\zeta_{j, o, p}$ is the charge contribution of species $j$ to plane $p$ of electrostatic surface $o, \Psi_{o, p}$, is the electric potential of the $p^{\text {th }}$ plane of the $o^{\text {th }}$ electrostatic surface and $R$ is the ideal gas constant [3]. Note that a surface species can be associated with multiple surface functional groups, but only one electrostatic surface.

2.4.2. Electrostatics of the surface. The formulation of the activity coefficient for electrostatic surfaces can be generalized as above, however, the determination of the charge and potential of the surface depends on the electrostatic model being employed. For a full description and comparison of different surface chemistry models consult [12], which is the text from which the constitutive relationships implemented here are pulled.

The simplest model is the constant capacitance model. The mineral surface is the only layer in this model. The charge of the mineral surface, $\sigma$, is calculated as the linear combination of charged species which reside on the surface

$$
\sigma_{o, p=1}=\frac{F}{S_{o} a_{o}} \sum_{j=1}^{n_{c}} c_{j} \zeta_{j, o, p=1}
$$

where $\sigma_{o, p=1}$ is the charge density of the mineral surface of the $o^{\text {th }}$ electrostatic surface (which is a constant capacitance surface), and $S_{o}$ and $a_{o}$ are the specific surface area and slurry density of electrostatic surface $o$. Note the charge contribution of a species to an electrostatic surface on which it does not reside will be zero.

The constant capacitance model simulates the mineral-liquid interface as a capacitor. The potential is therefore determined by the capacitance density of the interface

$$
\Psi_{o, p=1}=\frac{\sigma_{o, p=1}}{C_{o, q=1}}
$$

where $C_{o, q=1}$ is the capacitance density of the $q^{\text {th }}$ layer of the $o^{\text {th }}$ electrostatic surface. Note that charge neutrality is not possible for a constant capacitance surface.

The triple layer model is a more accurate model which approximates the mineralliquid interface as three capacitors in series. The first two, starting from the mineral surface have a constant capacitance density, the outer most layer has a variable capacitance density as determined by the properties of the bulk solution, according to the Grahame equation. Just as in the constant capacitance model the charge of a plane is the linear summation of charged species that reside on the plane, while the charge density of the outer layer is determined by the Grahame equation. We have

$$
\sigma_{o, p}=\frac{F}{S_{o} a_{o}} \sum_{j=1}^{n_{c}} c_{j} \zeta_{j, o, p}
$$

for $p=1,2$ and

$$
\sigma_{o, 3}=-\left(8 \times 10^{3} R T I e_{o} e_{w}\right)^{1 / 2} \sinh \left(\frac{F \Psi_{o, 3}}{2 R T}\right)
$$


The charge-potential relationship for the triple layer surface is then determined by

$$
\begin{aligned}
& \sigma_{o, p=1}=C_{o, q=1}\left(\Psi_{o, p=1}-\Psi_{o, p=2}\right) \\
& \sigma_{o, p=3}=C_{o, q=2}\left(\Psi_{o, p=3}-\Psi_{o, p=2}\right)
\end{aligned}
$$

Finally the triple layer surface must be charge neutral

$$
0=\sum_{p=1}^{n_{p}} \sigma_{o, p} .
$$

2.4.3. Basic stern model and diffuse layer model. These two models are limiting cases of the triple layer model. The Basic stern model is achieved when there is no sorption on the $p=2$ plane, and thus the potential drop across the $q=2$ layer is negligible. This is approximated by disallowing sorption reactions to occur on the $p=2$ plane and setting the capacitance density of the $q=2$ layer to a very large number (say 1000 coulombs $/ \mathrm{m}^{2}$ ). The diffuse layer model approximates the mineral-liquid interface as simply the diffuse ion cloud. This is achieved by ignoring the two inner constant capacitance layers of the triple layer by disallowing sorption on the $p=2$ plane, and setting the capacitance density of the inner two layers to a large number as before.

2.5. Practical Implementation. Given conventions within the geochemical community, there is some subtlety within the above formulation which requires detailing.

Primary among these is local chemical equilibrium, an assumption that is implemented in the geochemical code. Such an assumption is largely accepted for aqueous reaction. However, the formation of solids is a time dependent problem. In addition, formation/removal of solid and other phases requires a volume change. Thus the density of the phases are required. thus the present solver provides the saturation index, rather than mass or volume of solid. In future releases we hope to address this issue.

In addition, the quantity, $\mathrm{N}$, of a species has different unit conventions depending on the context. All aqueous species throughout the mathematical formulation are given in mole $/ \mathrm{m}^{3}$. Surface species however, have units of mole $/ \mathrm{m}^{3}$ in the composition matrix, whereas in the reaction matrix the mole fraction convention is adopted. This is due to complications in defining the reaction constant for reactions with multi-dentate species [11].

\section{Newton SOlver STRATEGIES}

3.1. A log-log formulation of the residual equations. Logarithm transformations of the concentrations and residual equations are certainly commonly used in Newton solver for chemical equations, see for example the recent paper [5]. For the concentrations, which are the unknowns in the system, using their logarithms as primary variables are the clear advantage of imposing in a soft way the positivity of the concentrations. For the residual equations, it seems natural to apply the logarithm to the reaction equations as they get linear, see (13a). However, to take the logarithm of the constitutive equations, that is replace in the Newton algorithm $\left[V^{t} x\right]_{i}=X_{i}$ with $\ln \left(V_{i}^{t} X\right)=\ln \left(X_{i}\right)$, seems less obvious. For example, the authors in [5] do not mention this choice. In [10], the authors, motivated by results from geometric programming, advocate for such treatment of the residual equations. 
We use the same notations as in the first section. Assuming that all components have activities equal to their concentrations, the chemical equilibrium reactions are given by

$$
\prod_{j=1}^{n_{c}} x_{j}^{a_{k, j}}=K_{k},
$$

for $k=1, \ldots, n_{e}$. We follow the approach of [10] and do not assume that the coefficients in $V$ are positive. We rewrite the conservation equations given by (3) as

$$
\sum_{j=1}^{n_{c}} V_{j, k}^{+} x_{j}-\sum_{j=1}^{n_{c}} V_{j, k}^{-} x_{j}=M_{k}
$$

where $V_{j, k}^{ \pm}$denotes the positive and negative par of $V_{j, k}$, so that $V_{j, k}=V_{j, k}^{+}-V_{j, k}^{-}$. Here $M_{k}$ is a given constant. We decompose $M_{k}$ as $M_{k}=M_{k}^{+}-M_{k}^{-}$, where $M_{k}^{+}=\max \left(0, M_{k}\right)$ and $M_{k}^{-}=-\min \left(0, M_{k}\right)$ so that either $M_{k}^{+}$or $M_{k}^{-}$are in fact zero. We rewrite these two sets of equations in the synthetic form

$$
f_{i}(x)=1 .
$$

In particular for $k=1, \ldots, n_{c}-n_{e}$, we have

$$
f_{k+n_{c}-n_{e}}(x)=\frac{\sum_{j=1}^{n_{c}}\left(V_{j, k}^{+} x_{j}\right)+M_{k}^{-}}{\sum_{j=1}^{n_{c}}\left(V_{j, k}^{-} x_{j}\right)+M_{k}^{+}}
$$

The geometric programming approach consists of introducing the logarithm variables $\hat{x}_{j}=\ln \left(x_{j}\right)$ and to solve, using Newton iterations, the residual equations

$$
\ln \left(f_{i}(x)\right)=0,
$$

i.e., $\ln \left(f_{i}\left(e^{\hat{x}_{1}}, \ldots, e^{\hat{x}_{n}}\right)\right)=0$. Let us briefly the approach of [10]. We compute the derivative of $\ln f_{k+n_{c}-n_{e}}$ with respect to $\hat{x}_{i}$ and we obtain

$$
\begin{aligned}
\frac{\partial}{\partial \hat{x}_{i}} \ln f_{k+n_{c}-n_{e}} & =\frac{\partial}{\partial \hat{x}_{i}} \ln \left(\sum_{j=1}^{n} V_{j, k}^{+} x_{j}+M_{k}^{-}\right)-\frac{\partial}{\partial \hat{x}_{i}} \ln \left(\sum_{j=1}^{n} V_{j, k}^{+} x_{j}+M_{k}^{-}\right) \\
& =\left(\frac{V_{i, k}^{+}}{\sum_{j=1}^{n} V_{j, k}^{+} x_{j}+M_{k}^{-}}-\frac{V_{i, k}^{-}}{\sum_{j=1}^{n} V_{j, k}^{-} x_{j}+M_{k}^{+}}\right) \frac{d x_{i}}{d \hat{x}_{i}} \\
& =\frac{V_{i, k}^{+} x_{i}}{\sum_{j=1}^{n} V_{j, k}^{+} x_{j}+M_{k}^{-}}-\frac{V_{i, k}^{-} x_{i}}{\sum_{j=1}^{n} V_{j, k}^{-} x_{j}+M_{k}^{+}}
\end{aligned}
$$

because $\frac{d x_{i}}{d \hat{x}_{i}}=x_{i}$. We denote by $W_{j, k}$ the right-hand side in (34). It turns out that $f_{k+n_{c}-n_{e}}$ can be rewritten as

$$
f_{k+n_{c}-n_{e}}=D_{k} \prod_{W_{j, k} \neq 0}\left(\frac{V_{j, k} x_{j}}{W_{j, k}}\right)^{W_{j, k}}
$$

where the constant $D_{k}$ is defined as

$$
D_{k}= \begin{cases}\left(\frac{M_{k}^{+}}{W_{k}^{+}}\right)^{W_{k}^{+}} & \text {if } M_{k}^{+}>0, \\ \left(\frac{M_{k}^{-}}{W_{k}^{-}}\right)^{W_{k}^{-}} & \text {if } M_{k}^{-}>0 .\end{cases}
$$


The residual equations as given by (35) take the form of a geometric programming problem. In such case, explicit expression for the inverse of the Jacobian are available. We refer to [10] for more details on this aspect which is not our prime interest.

We are more interested in the efficiency of the algorithm, which is based on the convexity of the residual equations. In [10], the authors even claim that, because of the convexity of every residual equation, the algorithm is unconditionally convergent. We are unsure about this claim and have not found in the literature evidence supporting it, but convexity of the residual equations can be expected to bring stability. When the coefficients of $V$ are positive, the residual equation given in (32) takes the form

$$
g(x)=\sum_{j=1}^{n} \alpha_{j} x_{j}
$$

for positive coefficients $\alpha_{j}$. Function of the form above retain their convexity when changing to logarithmic variables. We detail the proof since it is not done in [10]. The Hessian of $\ln (g)$ with respect to $\hat{x}$, which we denote $H$, is given by

$$
H_{l, j}=\frac{\partial}{\partial \hat{x}_{l} \partial \hat{x}_{i}} \ln (g)= \begin{cases}-g^{-2} \alpha_{i} x_{i} \alpha_{l} x_{l} & \text { if } i \neq l \\ -g^{-2} \alpha_{i}^{2} x_{i}^{2}+g^{-1} \alpha_{i} x_{i} & \text { if } i=l\end{cases}
$$

Hence, for any $u \in \mathbb{R}^{n}$, we have

$$
u^{t} H u=-|v|^{2}+u \cdot v
$$

for $v \in \mathbb{R}^{n}$ defined as

$$
v_{i}=\frac{\alpha_{i} x_{i} u_{i}}{g}
$$

It follows that $u_{i}-v_{i}=\left(\frac{g}{\alpha_{i} x_{i}}-1\right) v_{i}$ and therefore

$$
u^{t} H u=\sum_{i=1}^{n}\left(\frac{\sum_{j=1}^{n} \alpha_{j} x_{j}}{\alpha_{i} x_{i}}-1\right) v_{i}^{2}=\sum_{i=1}^{n} \sum_{\substack{j \neq i \\ j=1}}^{n} \frac{\alpha_{j} x_{j} \alpha_{i} x_{i} u_{i}^{2}}{g^{2}} \geq 0
$$

so that $\ln (g)$ is convex.

3.2. Apriori bounds based on conservation of constitutive elements. Let us denote by $X \in \mathbb{R}^{n_{c}-n_{e}}$ the concentration of each of the constitutive elements. When $V$ is given by the composition matrix, the vector $X$ corresponds to $M$ in (7). Thus, using the notations of the previous section, we have

$$
V^{t} x=X
$$

The coefficients of $V$ and $x$ are positive. Therefore, for each specie $i=\left\{1, \ldots, n_{c}\right\}$, we get

$$
x_{i} V_{i j} \leq X_{j},
$$

for all $j \in\left\{1, \ldots, n_{c}-n_{e}\right\}$. Given the total concentrations $X_{j}$, we end up with the following upper bound for $x_{i}$,

$$
x_{i} \leq \min _{j \in\left\{1, \ldots, n_{c}-n_{e}\right\}} \frac{X_{j}}{V_{i j}}=m_{i} .
$$

This simple bound turns out to improve significantly the robustness of the method, as shown in particular in the first numerical test below. They are used for chopping: If, after a Newton update, $x_{i}>m_{i}$, then we set $x_{i}=m_{i}$. 


\section{Transport Equations}

We consider the general case introduced in the first section but now the chemical species are transported. We denote by $u_{i}$ the flux of each component species. The governing equations are then

$$
\frac{\partial N_{i}}{\partial t}+\nabla \cdot\left(u_{i}\right)=F_{i}(N)
$$

where the source term $F_{i}$ is defined in (5). Using that $V^{t} F=0$, we obtain two set of equations from (38)

$$
\frac{\partial M}{\partial t}+\nabla \cdot\left(V^{t} u\right)=0
$$

and

$$
\frac{\partial P}{\partial t}+\nabla \cdot\left(W^{t} u\right)=W^{t} A G
$$

where $M$ and $P$ are defined in (7). If we assume that the time scale for the chemical equations is much faster than the time scale for transport in (40), then we obtain $W^{t} A^{t} G=0$, which is equivalent to $G=0$. The governing equations are therefore

$$
\begin{aligned}
\frac{\partial X}{\partial t}+\nabla \cdot\left(V^{t} u(x)\right) & =0, \\
G(x) & =0, \\
V^{t} x & =X .
\end{aligned}
$$

The flux $u_{i}(x)$ of each species is in the case of a single fluid phase given by

$$
u_{i}(x)= \begin{cases}x_{i} U & \text { if the component } i \text { is a dissolved component } \\ 0 & \text { if the component } i \text { belongs to the solid phase }\end{cases}
$$

Above $U$ denotes the fluid phase velocity. For a porous media with permeability $K$ and an incompressible fluid, $U$ satisfies $\nabla \cdot U=0$ and $U=-K \nabla p$.

\section{Test Cases}

5.1. Alkalinity-pH equation. To analyze the performance of the chemical solver, we use the test case presented in [8]. The aim is to compute the composition of sea water for a given alkalinity. The method used by Munhoven is very different from ours: Instead of solving directly the full system of equations, the system is reduced analytically to a scalar equation with one unknown, the $\mathrm{H}^{+}$concentration. Then, the equation takes the form of a third order polynomial and robust algorithms to solve this polynomial are derived, which also include apriori bounds for the roots. The resulting algorithms are very effective. However, the method is inherently tailored to this particular chemical system and activities are not included. The system includes 18 chemical species given by

$$
\begin{array}{ll}
\mathrm{H}_{2} \mathrm{CO}_{3}, \mathrm{HCO}_{3}^{-}, \mathrm{CO}_{3}^{2-}, & \mathrm{H}_{2} \mathrm{O}, \mathrm{H}^{+}, \mathrm{OH}^{-}, \\
\mathrm{B}(\mathrm{OH})_{4}^{-}, \mathrm{B}(\mathrm{OH})_{3}, & \mathrm{H}_{3} \mathrm{PO}_{4}, \mathrm{H}_{2} \mathrm{PO}_{4}^{-}, \mathrm{HPO}_{4}^{2-}, \mathrm{PO}_{4}^{3-}, \\
\mathrm{H}_{4} \mathrm{SiO}_{4}, \mathrm{H}_{3} \mathrm{SiO}_{4}^{-}, & \mathrm{HSO}_{4}^{-}, \mathrm{SO}_{4}^{2-}, \\
\mathrm{HF}, \mathrm{F}^{-} . &
\end{array}
$$


They are involved in the following chemical equations,

$$
\begin{aligned}
& \mathrm{H}_{2} \mathrm{CO}_{3} \stackrel{K_{C 1}}{\longleftrightarrow} \mathrm{H}^{+}+\mathrm{HCO}_{3}^{-}, \\
& \mathrm{HCO}_{3}^{-} \stackrel{K_{C 2}}{\longleftrightarrow} \mathrm{H}^{+}+\mathrm{CO}_{3}^{2-}, \\
& \mathrm{H}_{2} \mathrm{O} \stackrel{K_{w}}{\longleftrightarrow} \mathrm{H}^{+}+\mathrm{OH}^{-}, \\
& \mathrm{B}(\mathrm{OH})_{4}^{-} \stackrel{K_{B}}{\longleftrightarrow} \mathrm{B}(\mathrm{OH})_{3}+\mathrm{OH}^{-}, \\
& \mathrm{H}_{3} \mathrm{PO}_{4} \stackrel{K_{P 1}}{\longleftrightarrow} \mathrm{H}^{+}+\mathrm{H}_{2} \mathrm{PO}_{4}^{-}, \\
& \mathrm{H}_{2} \mathrm{PO}_{4}^{-} \stackrel{K_{P 2}}{\longleftrightarrow} \mathrm{H}^{+}+\mathrm{HPO}_{4}^{2-}, \\
& \mathrm{HPO}_{4}^{2-} \stackrel{K_{P 3}}{\longleftrightarrow} \mathrm{H}^{+}+\mathrm{PO}_{4}^{3-}, \\
& \mathrm{H}_{4} \mathrm{SiO}_{4} \stackrel{K_{S i}}{\longleftrightarrow} \mathrm{H}^{+}+\mathrm{H}_{3} \mathrm{SiO}_{4}^{-}, \\
& \mathrm{HSO}_{4}^{-} \stackrel{K_{S O 4}}{\longleftrightarrow} \mathrm{H}^{+}+\mathrm{SO}_{4}^{2-}, \\
& \mathrm{HF}_{K_{F}}^{\longleftrightarrow} \mathrm{H}^{+}+\mathrm{F}^{-} .
\end{aligned}
$$

The total alkalinity is given in this case by

$$
\begin{aligned}
\mathrm{Alk}_{\mathrm{T}}= & {\left[\mathrm{HCO}_{3}^{-}\right]+2\left[\mathrm{CO}_{3}^{2-}\right] } \\
& +\left[\mathrm{B}(\mathrm{OH})_{4}-\right]+\left[\mathrm{OH}^{-}\right]+\left[\mathrm{HPO}_{4}^{2-}\right]+2\left[\mathrm{PO}_{4}^{3-}\right]+\left[\mathrm{H}_{3} \mathrm{SiO}_{4}-\right] \\
& \quad-\left[\mathrm{H}^{+}\right]-\left[\mathrm{HSO}_{4}-\right]-[\mathrm{HF}]-\left[\mathrm{H}_{3} \mathrm{PO}_{4}\right] .
\end{aligned}
$$

We use the routines provided in SolveSAPHE, which is available in the supplementary material of [8], to compute the chemical kinetic constants at temperature $T=275.15 \mathrm{~K}$, pressure $p=0$ bar and salinity coefficient $s=35$. We have

\begin{tabular}{rrrrrrrrrr}
$p K_{C_{1}}$ & $p K_{C_{2}}$ & $p K_{B}$ & $p K_{P_{1}}$ & $p K_{P_{2}}$ & $p K_{P_{3}}$ & $p K_{S i}$ & $p K_{S O 4}$ & $p K_{F}$ & $p K_{w}$ \\
\hline 6.1 & 9.3 & 8.9 & 1.6 & 6.2 & 9.3 & 9.8 & 0.58 & 2.5 & 14
\end{tabular}

The constant inputs are given by

\begin{tabular}{cccccc} 
& {$[\mathrm{F}]_{\mathrm{T}}$} & {$\left[\mathrm{SO}_{4}\right]_{\mathrm{T}}$} & {$[\mathrm{B}]_{\mathrm{T}}$} & {$[\mathrm{P}]_{\mathrm{T}}$} & {$\left[\mathrm{SiO}_{4}\right]_{\mathrm{T}}$} \\
\hline moll $^{-1}$ & 0.068 & 28 & 0.42 & $5 \mathrm{e}-7$ & $5 \mathrm{e}-6$
\end{tabular}

Note that the total concentrations of $\mathrm{P}$ and $\mathrm{Si}$ that are given in [8] differ from the one from the code which is provided in the supplementary online material. We consider the values given in the code which are consistent with the results that are shown in the paper. We look at the test case SW2 from [8]. We compute the chemical composition of the mixture for values of $[\mathrm{C}]_{\mathrm{T}}$ ranging from 1.85 to $3.35 \mathrm{mmol} \mathrm{kg}^{-1}$ and values of $\left[\mathrm{Alk}_{\mathrm{T}}\right]$ ranging from 2.2 to $3.5 \mathrm{mmol} \mathrm{kg}{ }^{-1}$. We use a resolution of $50 \times 50$. The values for the $\mathrm{pH}$ are given in Figure 1 and they correspond to the values obtained in [8].

In Figure 3, we plot the number of Newton iterations necessary for convergence. The convergence criteria in this case is $\left|\left[\mathrm{H}^{+}\right]^{n+1}-\left[\mathrm{H}^{+}\right]^{n}\right| /\left[\mathrm{H}^{+}\right]^{n}<\varepsilon$, and we use $\varepsilon=1 \times 10^{-8}$, as in [8]. We consider two cases for different choices of the initial guess. In the first case, we use an educated initial guess, based on the physical bounds on the unknowns. In the second case, the initial guess is set uniformly to 


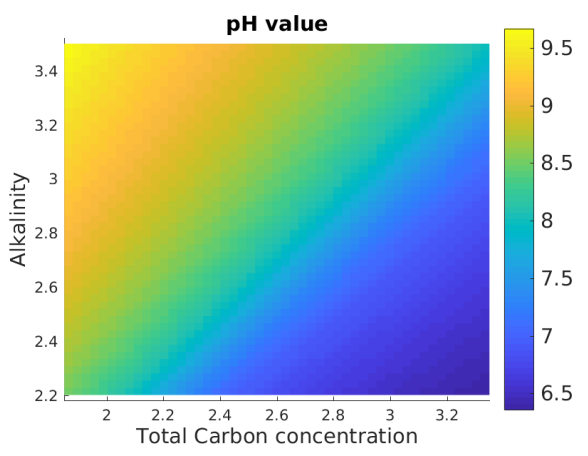

Figure 1. plot of $\mathrm{pH}$ values

$1 \mathrm{moll}^{-1}$ for all the unknown. In both cases, the method always converges and the second case uses only a few extra iterations more than the first case, showing the robustness of the approach.
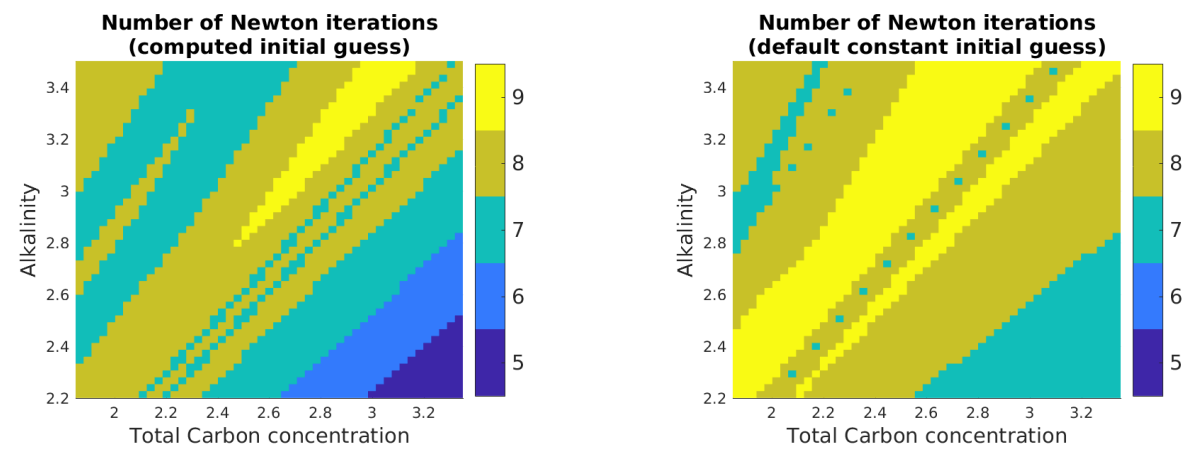

Figure 2. Number of Newton iterations. We observe that the number of iterations is smaller for the computed initial guess. However, even for rough uniform initial guess, the method always converges.

We use this test case to assess the importance of the physical bound in the convergence of the Newton algorithm. To do so, we switch off the chopping of the variables after each Newton updates. The results are presented in Figure 3. The method is not always convergent and require otherwise significantly more Newton steps. This result shows the important of the chopping step.

5.2. Equilibrium with an electrostatic surface. We consider here an amphoteric surface with the sorption of $\mathrm{H}^{+}, \mathrm{Na}^{+}$, and $\mathrm{Cl}^{-}$. We have one group site which we denote SO, being an arbitrary hydroxide surface, which can evolve as the following species

$$
>\mathrm{SOH},>\mathrm{SO}^{-},>\mathrm{SOH}_{2}^{+},>\mathrm{SONa},>\mathrm{SOH}_{2} \mathrm{Cl},
$$

depending on the sorbed component. We use the triple layer model to compute the equilibrium of this system. We use the same notation as in the section above where 


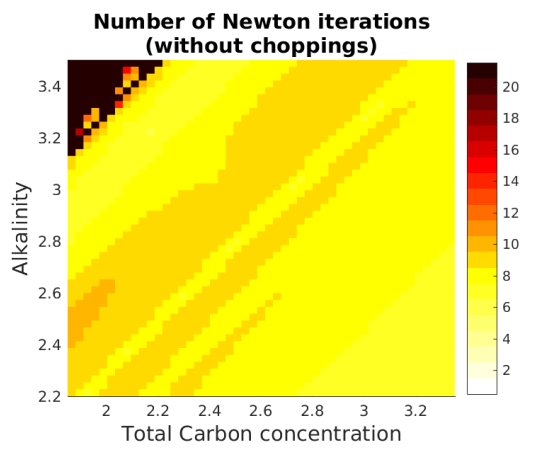

Figure 3. Number of Newton iterations in the case where the chopping of the variables is not used. For some of the parameters, which corresponds to the value above 21 in the plot, the method does not converge.

the triple layer model is introduced but, since there is only a single surface, we drop the index $o$. Each surface component will contribute to the charge of a given layer. In this example, $\mathrm{H}^{+}$sorbs directly to the mineral surface, and is called an inner sphere sorption complex. The species $\mathrm{Na}^{+}$and $\mathrm{Cl}^{-}$are geometrically limited, and can not sorb directly to this mineral surface. Thus they occupy the second layer $(p=2)$ and contribute their charge to $p=2$. Therefore, the charged components $>\mathrm{SO}^{-}$and $>\mathrm{SOH}_{2}^{+}$contribute their charge to the first layer. The non-charged component may contribute through their polarization as they spread across the two first layer. In this case, $>\mathrm{SoNa}$ contributes with a negative charge layer to the first layer and a positive charge to the second layer. For $>\mathrm{SOH}_{2} \mathrm{Cl}$, it is the opposite. The component $>\mathrm{SOH}$ is inactive in this context, see Figure 4 for a table overview.

The unknowns are the species concentrations, $(45)$

$>\mathrm{SOH},>\mathrm{SO}^{-},>\mathrm{SOH}_{2}^{+},>\mathrm{SONa},>\mathrm{SOH}_{2} \mathrm{Cl}, \mathrm{H}^{+}, \mathrm{OH}^{-}, \quad \mathrm{H}_{2} \mathrm{O}, \quad \mathrm{Na}^{+}, \mathrm{Cl}^{-}, \quad \mathrm{NaCl}$

and the corresponding activities, the potentials and charges of the layers $\left(\Psi_{i}, \sigma_{i}\right.$, for $i=1,2,3)$ and the ionic strength $I$. We index the species using the same ordering as in (45). We denote by $x_{s}$ the concentration of the surface species $\left(x_{s, i}=x_{i}\right.$ for $i=1, \ldots, 5)$ and $x_{a}$ for the aqueous species $\left(x_{a, i}=x_{i+5}\right.$ for $\left.i=1, \ldots, 6\right)$. The total number of unknowns is equal to 29 .

Let us present the governing equations. The chemical equilibrium equations are

$$
A \hat{x}+A \hat{\gamma}=\hat{K},
$$

for $A \in \mathbb{R}^{n_{r} \times n_{c}}$, see Figure 5. the definition of the activities for the aqueous species follow from (22),

$$
\hat{\gamma}_{\mathrm{NaCl}}=\hat{\gamma}_{\mathrm{H}_{2} \mathrm{O}}=0 \quad \text { and } \quad \hat{\gamma}_{\mathrm{H}^{+}}=-\hat{\gamma}_{\mathrm{OH}}=\hat{\gamma}_{\mathrm{Na}^{+}}=-\hat{\gamma}_{\mathrm{Cl}^{-}}=f(I)
$$

where the definition of the function $f$ can be inferred from (22). The equation for the ionic strength is linear and given by (23), which we rewrite as

$$
I=\kappa^{t} x_{a}
$$


for $\kappa=(1,-1,0,1,-1,0)^{t}$ in this case. The equations for the activities of the surface species are given by $(24)$, which we rewrite as

$$
\hat{\gamma}_{s}=c_{1} B \Psi
$$

for a matrix $B \in \mathbb{R}^{n_{s} \times 3}$. Our goal here is to present the form of the equations and to simplify the notations we denote generically by $c_{i}$ the constant physical terms that enter the equations. The defining equations for the charge are

$$
\sigma=c_{2} B^{t} x_{s}+G\left(\sinh \left(\frac{F \Psi_{3}}{2 R T}\right)\right),
$$

where $G$ is a function in $\mathbb{R}^{3}$ which is non-zero only for the third component. The capacitance relations are

$$
\sigma_{1}=C_{1}\left(\Psi_{1}-\Psi_{2}\right), \quad \sigma_{2}=C_{2}\left(\Psi_{3}-\Psi_{2}\right) .
$$

The charge balance is

$$
\sigma_{1}+\sigma_{2}+\sigma_{3}=0 .
$$

The governing equations are given by (46) and form a system of 24 equations. We have 5 master components, given by

$$
\mathrm{H}, \mathrm{O}, \mathrm{Na}, \mathrm{Cl},>\mathrm{SO},
$$

so that we match the 29 unknowns. Let us now set a numerical test. We vary

\begin{tabular}{|c|c|c|}
\hline reaction & \multicolumn{2}{|c|}{ equilibrium constant } \\
\hline \multirow{6}{*}{$\begin{array}{l}>\mathrm{SOH} \rightleftarrows>\mathrm{SO}^{-}+\mathrm{H}^{+}, \\
>\mathrm{SOH}+\mathrm{H}^{+} \rightleftarrows>\mathrm{SOH}_{2}^{+}, \\
>\mathrm{SO}^{-}+\mathrm{Na}^{+} \rightleftarrows>\mathrm{SONa}^{+} \\
>\mathrm{SOH}_{2}^{+}+\mathrm{Cl}^{-} \rightleftarrows>\mathrm{SOH}_{2} \mathrm{Cl}, \\
\mathrm{H}_{2} \mathrm{O} \rightleftarrows \mathrm{H}^{+}+\mathrm{OH}^{-}, \\
\mathrm{NaCl} \rightleftarrows \mathrm{Na}^{+}+\mathrm{Cl}^{-},\end{array}$} & & $10^{-7.5} \mathrm{moll}^{-1}$ \\
\hline & & $10^{2}(\mathrm{~mol} / \mathrm{l})^{-1}$ \\
\hline & & $0^{-1.9}(\mathrm{~mol} / \mathrm{l})^{-1}$ \\
\hline & & $10^{1}(\mathrm{~mol} / \mathrm{l})^{-1}$ \\
\hline & & $10^{-14} \mathrm{moll}^{-1}$ \\
\hline & & $10 \mathrm{moll}^{-1}$ \\
\hline \multicolumn{3}{|c|}{ layer 1} \\
\hline$>\mathrm{SOH}:$ & 0 & 0 \\
\hline$>\mathrm{SO}^{-}:$ & -1 & 0 \\
\hline$>\mathrm{SOH}_{2}^{+}:$ & 1 & 0 \\
\hline$>$ SONa: & -1 & 1 \\
\hline$>\mathrm{SOH}_{2} \mathrm{Cl}:$ & 1 & -1 \\
\hline
\end{tabular}
the total hydrogen concentration $[\mathrm{H}]_{T}$ from $1 \times 10^{-4}$ to $1 \times 10^{-10} \mathrm{moll}^{-1}$. For the master component $\mathrm{Na}$ and $\mathrm{Cl}$, we use constant total concentrations given by $[\mathrm{Na}]_{T}=[\mathrm{Cl}]_{T}=1 \times 10^{-2} \mathrm{moll}^{-1}$. The normalized water concentration is also kept constant and equal to $1 \mathrm{moll}^{-1}$.

FIGURE 4. Reaction and surface species contribution

The potential and charge of each layer of the chemical system are plotted in figure 6 . It can be seen that these quantities vary smoothly as a function of $\mathrm{pH}$, 


$$
A=\left(\begin{array}{ccccccccccc}
1 & 1 & 0 & 0 & 0 & -1 & 0 & 0 & 0 & 0 & 0 \\
0 & 0 & 1 & 1 & -1 & 0 & 0 & 0 & 0 & 0 & 0 \\
1 & 0 & 0 & 0 & 0 & 0 & 1 & -1 & 0 & 0 & 0 \\
-1 & 0 & 0 & 0 & 0 & 0 & 0 & -1 & 1 & 0 & 0 \\
0 & 0 & -1 & 0 & 0 & 0 & -1 & 0 & 0 & 1 & 0 \\
0 & 0 & 0 & -1 & 0 & 0 & 0 & 0 & -1 & 0 & 1
\end{array}\right) \quad B^{t}=\left(\begin{array}{ccc}
0 & 0 & 0 \\
-1 & 0 & 0 \\
1 & 0 & 0 \\
-1 & 1 & 0 \\
1 & -1 & 0
\end{array}\right)
$$

FiguRE 5. Matrices involved in this test case
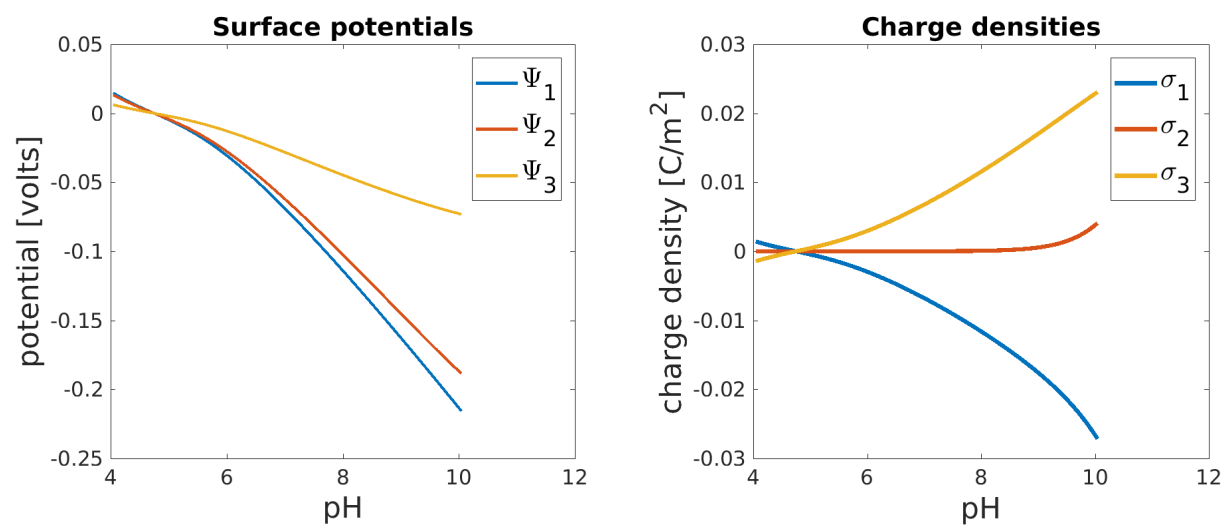

Figure 6. Plots of the surface potential and charge densities as a function of the solution $\mathrm{pH}$

which is expected. Further, the charge of each layer sums to zero, as is enforced in the model. Finally, it can be seen that near $\mathrm{pH}=3$ the potential and charge of all layers is zero. This is known as the point of zero charge and is a defining characteristic of an amphoteric surface. 


\section{ACKNOWLEDGMENT}

X. Raynaud thanks support from the Norwegian Research Council (KPN 280651). This work is funded in part by the Center for Frontiers of Subsurface Energy Security, an Energy Frontier Research Center funded by the U.S. Department of Energy, Office of Science, Basic Energy Sciences under Award \#de-sc0001114.

\section{REFERENCES}

[1] C. W. Davies. Ion association. Butterworths, Washington, 1962.

[2] George L. Gaines and Henry C. Thomas. Adsorption studies on clay minerals. ii. a formulation of the thermodynamics of exchange adsorption. The Journal of Chemical Physics, 21(4):714$718,1953$.

[3] T. Hiemstra, J.C.M. De Wit, and W H Van Riemsdijk. Multisite Proton Adsorption Modeling at the Solid / Solution Interface of (Hydr) oxides : A New Approach. Journal of colloid and interface science, 133(1):105-116, 1989.

[4] Stein Krogstad, Knut-Andreas Lie, Olav Møyner, Halvor Møll Nilsen, Xavier Raynaud, Bård Skaflestad, et al. Mrst-ad-an open-source framework for rapid prototyping and evaluation of reservoir simulation problems. In SPE reservoir simulation symposium. Society of Petroleum Engineers, 2015.

[5] Haishan Luo, Emad W Al-Shalabi, Mojdeh Delshad, Krishna Panthi, Kamy Sepehrnoori, et al. A robust geochemical simulator to model improved oil recovery methods. In SPE Reservoir Simulation Symposium. Society of Petroleum Engineers, 2015.

[6] CG Malmberg and AA Maryott. Dielectric constant of water from 00 to 1000 c. Journal of research of the National Bureau of Standards, 56(1):1-8, 1956.

[7] Colin McNeece, Xavier Raynaud, Marc Hesse, and Halvor Møll Nilsen. matlab geochemisry (match): a tool for the solution of equilibrium for geochemical system. 2017. https://bitbucket.org/mrst/matlab-geochemistry.

[8] G Munhoven. Mathematics of the total alkalinity-ph equation-pathway to robust and universal solution algorithms: the solvesaphe package v1. 0.1, geosci. model dev., 6, 1367-1388, 2013.

[9] David L Parkhurst, CAJ Appelo, et al. Description of input and examples for phreeqc version 3-a computer program for speciation, batch-reaction, one-dimensional transport, and inverse geochemical calculations. 2013

[10] Thomas Wayne Wall, Doran Greening, and R. E. D. Woolsey. Solving complex chemical equilibria using a geometric-programming based technique. Operations Research, 34(3):345$355,1986$.

[11] Zimeng Wang and Daniel E Giammar. Mass action expressions for bidentate adsorption in surface complexation modeling: Theory and practice. Environmental science 85 technology, 47(9):3982-3996, 2013.

[12] John Westall and Herbert Hohl. A comparison of electrostatic models for the oxide/solution interface. Advances in Colloid and Interface Science, 12(4):265-294, 1980. 